\title{
SDSS J074511.56+194926.5: DISCOVERY OF A METAL-RICH AND TIDALLY DISTORTED EXTREMELY LOW MASS WHITE DWARF
}

\author{
A. Gianninas ${ }^{1}$, J. J. Hermes ${ }^{2,3}$, Warren R. Brown ${ }^{4}$, P. Dufour ${ }^{5}$, Sara D. Barber ${ }^{1}$, Mukremin Kilic ${ }^{1}$, \\ ScotT J. KenYon ${ }^{4}$, And SAMuel T. HARROLD ${ }^{2}$ \\ ${ }^{1}$ Homer L. Dodge Department of Physics and Astronomy, University of Oklahoma, \\ 440 W. Brooks St., Norman, OK 73019, USA; alexg@nhn.ou.edu \\ ${ }^{2}$ Department of Astronomy, University of Texas at Austin, Austin, TX 78712, USA \\ ${ }^{3}$ Department of Physics, University of Warwick, Coventry CV4 7AL, UK \\ ${ }^{4}$ Smithsonian Astrophysical Observatory, 60 Garden St., Cambridge, MA 02138, USA \\ ${ }^{5}$ Département de Physique, Université de Montréal, C.P. 6128, Succ. Centre-Ville, Montréal, Québec H3C 3J7, Canada \\ Received 2013 August 29; accepted 2013 December 5; published 2014 January 15
}

\begin{abstract}
We present the discovery of an unusual, tidally distorted extremely low mass white dwarf (WD) with nearly solar metallicity. Radial velocity measurements confirm that this is a compact binary with an orbital period of $2.6975 \mathrm{hr}$ and a velocity semi-amplitude of $K=108.7 \mathrm{~km} \mathrm{~s}^{-1}$. Analysis of the hydrogen Balmer lines yields an effective temperature of $T_{\text {eff }}=8380 \mathrm{~K}$ and a surface gravity of $\log g=6.21$ that in turn indicate a mass of $M=0.16 M_{\odot}$ and a cooling age of $4.2 \mathrm{Gyr}$. In addition, a detailed analysis of the observed metal lines yields abundances of $\log$ $(\mathrm{Mg} / \mathrm{H})=-3.90, \log (\mathrm{Ca} / \mathrm{H})=-5.80, \log (\mathrm{Ti} / \mathrm{H})=-6.10, \log (\mathrm{Cr} / \mathrm{H})=-5.60$, and $\log (\mathrm{Fe} / \mathrm{H})=-4.50$, similar to the sun. We see no evidence of a debris disk from which these metals would be accreted, though the possibility cannot entirely be ruled out. Other potential mechanisms to explain the presence of heavy elements are discussed. Finally, we expect this system to ultimately undergo unstable mass transfer and merge to form a $\sim 0.3-0.6 M_{\odot}$ WD in a few Gyr.
\end{abstract}

Key words: binaries: close - stars: abundances - stars: individual (SDSS J074511.56+194926.5) - techniques: photometric - techniques: spectroscopic - white dwarfs

Online-only material: color figures

\section{INTRODUCTION}

Over the course of the past several years, we have successfully targeted extremely low mass (ELM) white dwarfs (WDs) as part of the ELM survey (Brown et al. 2010, 2012, 2013; Kilic et al. 2011, 2012a). These WDs are characterized by surface gravities of $5.0<\log g<7.0$ or, alternatively, with masses $M<0.25 M_{\odot}$. ELM WDs are necessarily the product of evolution within compact binary systems since the Galaxy is not yet old enough to produce them through single star evolution. This assertion is corroborated through radial velocity measurements, which confirm that all ELM WDs are in tight binary systems with periods $P_{\text {orb }} \leqslant 1$ day (Brown et al. 2013; Kilic et al. 2012a). ELM WDs are understood to be He-core WDs formed when the companion strips the outer envelope from a post-main-sequence star before the star reaches the tip of the red giant branch and ignites the helium.

ELM WD binaries afford us a unique opportunity to study the endpoint of the evolution of compact binaries. Furthermore, these systems could represent the eventual progenitors of Type Ia supernovae, underluminous.Ia supernovae (Bildsten et al. 2007), and AM CVn systems (Kilic et al. 2013c), as well as being strong sources of gravitational waves that result in orbital decay (Hermes et al. 2012b; Kilic et al. 2013a). Due to the nature of these close binaries, they also afford us the opportunity to study various phenomena that cause luminosity variations in the light curves of these stars. These include ellipsoidal variations due to tidal distortion, Doppler beaming, and eclipses by the companion (Hermes et al. 2012a, 2012b). The detection and analysis of these effects are important because they provide model-independent measurements of fundamental parameters such as the stellar radius and mass.
Another point of interest is the fact that the optical spectra of all ELM WDs with $\log g<6.0$ show the Ca K line at $3933 \AA$. Indeed, this phenomenon has been mentioned in most previous ELM papers but has not yet been explored in a quantitative fashion. In higher mass WDs, the presence of metallic absorption lines in their optical spectra is explained by the ongoing accretion of material from a circumstellar debris disk. These polluted WDs must be actively accreting material to explain the observed abundances since diffusion timescales for heavy elements in WD atmospheres are much shorter than their evolutionary timescales. This paradigm is supported by the detection of debris disks through infrared (IR) excess in the emission of more than two dozen WDs (Jura 2003; Kilic et al. 2006; Farihi et al. 2009; Barber et al. 2012). The heavy elements observed in the spectra of otherwise pure hydrogen or helium atmosphere WDs represent a means to study the composition of planetary bodies that survived the late phases of stellar evolution (Dufour et al. 2010; Klein et al. 2010, 2011; Zuckerman et al. 2007). There is growing evidence that perhaps all metal-polluted WDs have acquired their heavy material from an orbiting debris disk reservoir whose origin is explained by the tidal disruption of one or many large rocky bodies that ventured too close to the star (Debes \& Sigurdsson 2002; Farihi et al. 2010a, 2010b; Jura 2003, 2006, 2008; Jura et al. 2007; Melis et al. 2010). Such disks, which are easily detectable at IR wavelengths, are now being detected at an accelerated pace with more than 20 cases uncovered in the last $6 \mathrm{yr}$ alone (Farihi et al. 2009; Farihi 2011; Debes et al. 2012; Kilic et al. 2012b; Hoard et al. 2013, and references therein). Moreover, models that aim to explore and understand the actual accretion of the debris disk material onto the surface of the WD seem to be in good agreement with observations (Rafikov 2011a, 2011b; Rafikov \& Garmilla 2012). 


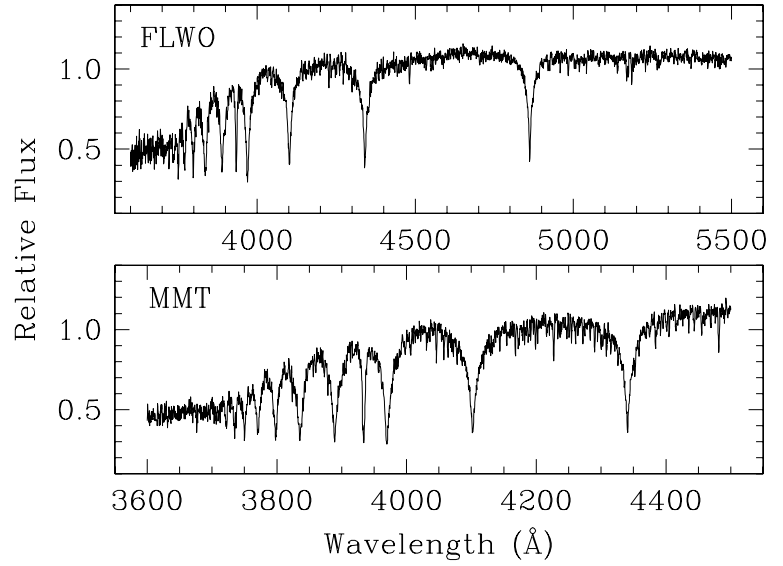

Figure 1. Radial-velocity-corrected and summed optical spectra of J0745+1949. The spectra are normalized to unity at $4200 \AA$. The lower resolution spectrum taken at FLWO (top) covers a range from 3600 to $5500 \AA$, while the spectrum obtained at the MMT (bottom) covers a smaller spectral range from 3600 to $4500 \AA$ A but at a higher resolution.

On the other hand, recent calculations show that some metals (e.g., C, Al, Si) can potentially be maintained in the atmospheres of WDs with $\log g=8.0$ and as cool as $T_{\text {eff }}=20,000 \mathrm{~K}$ by radiative forces (Chayer $\&$ Dupuis 2010). If we consider that ELM WDs have surface gravities two to three orders of magnitude weaker, radiative levitation may be relevant for maintaining metals in the atmospheres of these WDs. What is certain is that a detailed analysis of the metal abundances in ELM WDs is a necessary first step in understanding the origin of the metal pollutants in these stars. In this connection, the recent study of the WD companion of PSR J1816+4510 by Kaplan et al. (2013) is most intriguing. Their analysis yielded $T_{\text {eff }}=16,000 \pm 500 \mathrm{~K}$ and $\log g=4.9 \pm 0.3$ with super-solar abundances of $\mathrm{He}, \mathrm{Mg}, \mathrm{Si}, \mathrm{Ca}$ and $\mathrm{Fe}$.

In this paper, we present the detailed spectroscopic and photometric analysis of SDSS J074511.56+194926.5 (hereafter J0745), a new metal-rich and tidally distorted ELM WD in a compact binary. In Section 2 we present our spectroscopic and photometric observations. Next, Section 3 outlines the model atmospheres used in our spectroscopic analysis, and we present in Section 4 our analysis of all the available spectroscopic and photometric data. This is followed, in Section 5, by a discussion of the results and potential evolutionary scenarios that would apply to J0745. Finally, we make some concluding remarks and discuss future work in Section 6.

\section{OBSERVATIONS}

\subsection{Optical Spectroscopy}

J0745 was first observed on 2010 November 5 using the FLWO $1.5 \mathrm{~m}$ telescope and the FAST spectrograph (Fabricant et al. 1998). We used the 600 line $\mathrm{mm}^{-1}$ grating and a $1^{\prime \prime} .5$ slit, providing wavelength coverage from $3500 \AA$ to $5500 \AA$ and a spectral resolution of $1.7 \AA$. Initial stellar atmosphere fits revealed J0745 to be a candidate ELM WD. Follow-up spectra obtained one year later at the FLWO $1.5 \mathrm{~m}$ revealed a significant change in radial velocity. We thus obtained additional spectroscopy on five more nights to constrain J0745's orbital solution. All spectra were paired with a comparison lamp exposure and have an average $13 \mathrm{~km} \mathrm{~s}^{-1}$ radial velocity uncertainty.
Table 1

Available Broadband Photometry for J0745

\begin{tabular}{lcc}
\hline \hline Filter & $\begin{array}{c}\lambda_{\text {eff }} \\
(\mu \mathrm{m})\end{array}$ & Magnitude \\
\hline FUV & 0.153 & $22.321 \pm 0.380$ \\
NUV & 0.229 & $18.665 \pm 0.040$ \\
\hline$u$ & 0.355 & $17.426 \pm 0.016$ \\
$g$ & & $17.423 \pm 0.016$ \\
& 0.467 & $16.491 \pm 0.008$ \\
$r$ & & $16.505 \pm 0.010$ \\
& 0.617 & $16.488 \pm 0.018$ \\
$i$ & & $16.395 \pm 0.011$ \\
& & $16.479 \pm 0.011$ \\
$z$ & 0.748 & $16.458 \pm 0.013$ \\
& & $16.564 \pm 0.014$ \\
$J$ & 0.893 & $16.555 \pm 0.020$ \\
$H$ & & $15.915 \pm 0.058$ \\
$K$ & 1.241 & $16.156 \pm 0.171$ \\
$W 1$ & 1.651 & $15.655 \pm 0.172$ \\
\hline
\end{tabular}

We also obtained higher signal-to-noise $(\mathrm{S} / \mathrm{N})$ observations on 2012 May 23 with the $6.5 \mathrm{~m}$ MMT telescope and the Blue Channel spectrograph (Schmidt et al. 1989). We used the 832 line $\mathrm{mm}^{-1}$ grating and a 1".0 slit, providing wavelength coverage $3600 \AA$ to $4500 \AA$ and a spectral resolution of $1.0 \AA$. The individual MMT spectra have $7 \mathrm{~km} \mathrm{~s}^{-1}$ radial velocity uncertainty; the summed MMT spectrum has $\mathrm{S} / \mathrm{N}=120$ per resolution element. The summed FLWO and MMT spectra are shown in Figure 1. Though the FLWO spectrum provides better wavelength coverage, the MMT spectrum has a higher $\mathrm{S} / \mathrm{N}$ and a better resolution, allowing for a much better identification of all the observable metal lines. Consequently, the MMT spectrum will be the basis of our atmospheric parameter and metal abundance determinations.

\subsection{Time-series Photometry}

We obtained a total of $6.6 \mathrm{hr}$ of time-series photometry for J0745 using the Argos frame transfer camera mounted on the McDonald $2.1 \mathrm{~m}$ telescope (Nather \& Mukadam 2004). Observations were obtained over the course of two observing runs in 2012 November (4.9 hr) and 2013 March (1.7 hr). Our exposures ranged from 10 to $15 \mathrm{~s}$ with negligible read-out time and were obtained through a $3 \mathrm{~mm}$ BG40 filter to reduce sky noise.

We performed weighted, circular, aperture photometry on the calibrated frames using the external IRAF package $c c d \_h s p$ written by Antonio Kanaan (Kanaan et al. 2002). We divided the sky-subtracted light curves by the brightest comparison stars in the field to correct for transparency variations and applied a timing correction to each observation to account for the motion of the Earth around the barycenter of the solar system (Stumpff 1980; Thompson \& Mullally 2009). We have also reduced, in an identical manner, the bright nearby star SDSS J074508.34+194958.4. This star has a similar $g-r$ color as our target and helps us constrain the differential atmospheric effects on our time-series photometry.

\subsection{Broadband Photometry}

In Table 1, we list all of the available broadband photometry for J0745 from ultraviolet (UV), optical, and IR surveys. There 
are two available sets of ugriz magnitudes from the Sloan Digital Sky Survey (SDSS) Data Release 9 (Ahn et al. 2012). The first set, corresponding to the first of each pair of ugriz magnitudes listed in Table 1, was obtained on 2003 January 25, while the second set of observations were taken a week later on 2003 February 1. J0745 was also detected in the UV by the Galaxy Evolution Explorer (GALEX; Martin et al. 2005). In addition, IR photometry is available from the Two Micron All Sky Survey (2MASS; Skrutskie et al. 2006) and the Wide-field Infrared Survey Explorer (WISE; Wright et al. 2010). We only list the WISE magnitude in the $W 1$ band since the $\mathrm{S} / \mathrm{N}$ of the $W 2, W 3$, and $W 4$ band observations is $\mathrm{S} / \mathrm{N}<4$.

\section{MODEL ATMOSPHERES}

\subsection{Pure Hydrogen Model Atmospheres}

We use the hydrogen-rich model atmospheres described at length in Tremblay et al. (2010), and references therein, for the analysis of the hydrogen Balmer lines, from which we obtain our measurements of $T_{\text {eff }}$ and $\log g$. Briefly, these models assume a plane-parallel geometry, hydrostatic equilibrium, and local thermodynamic equilibrium (LTE). The assumption of LTE is justified as our model grid is restricted to $T_{\text {eff }}<30,000 \mathrm{~K}$, where NLTE effects are not yet significant. Furthermore, these models include the new Stark broadening profiles from Tremblay $\&$ Bergeron (2009) that include the occupation probability formalism of Hummer \& Mihalas (1988) directly in the line profile calculation. Our grid covers $T_{\text {eff }}$ from $4000 \mathrm{~K}$ to $30,000 \mathrm{~K}$ in steps ranging from 250 to $5000 \mathrm{~K}$, and $\log g$ from 5.0 to 9.5 in steps of 0.25 dex.

\subsection{Model Atmospheres with Metals}

We calculated metal-blanketed model atmospheres and synthetic spectra using the same code that was used to model the heavily metal polluted DBZ star J0738+1835 (Dufour et al. 2012). As a first step, we calculated a pure hydrogen model using the atmospheric parameters $\left(T_{\text {eff }}\right.$ and $\left.\log g\right)$ as determined from the fits to the Balmer lines. We then compared this model with an equivalent model computed using the code described in the previous section and confirmed that the thermodynamic structures were identical. Next, we proceeded to calculate several grids of synthetic spectra, one for each element of interest (i.e., $\mathrm{Mg}, \mathrm{Ca}, \mathrm{Ti}, \mathrm{Cr}$, and $\mathrm{Fe}$ ), keeping the abundance of all other elements fixed to their expected values (by assuming CI chondrite ratios from Lodders 2003). The individual grids cover a range of abundances from $\log [n(\mathrm{Z}) / n(\mathrm{H})]=-3.0$ to -10.0 , in steps of 0.5 dex. These grids are then used to determine the different elemental abundances.

\section{ANALYSIS}

\subsection{Atmospheric Parameters}

Our Balmer line fits use the so-called spectroscopic technique described in Gianninas et al. (2011) and references therein. One important difference between our procedure and that of Gianninas et al. (2011) is that we fit higher-order Balmer lines, up to and including H12, observed in low surface gravity ELM WDs. The higher-order Balmer lines are quite sensitive to $\log g$ and consequently improve our surface gravity measurement. Our error estimates combine the internal error of the model fits, obtained from the covariance matrix of the fitting algorithm, and the external error, obtained from multiple observations of

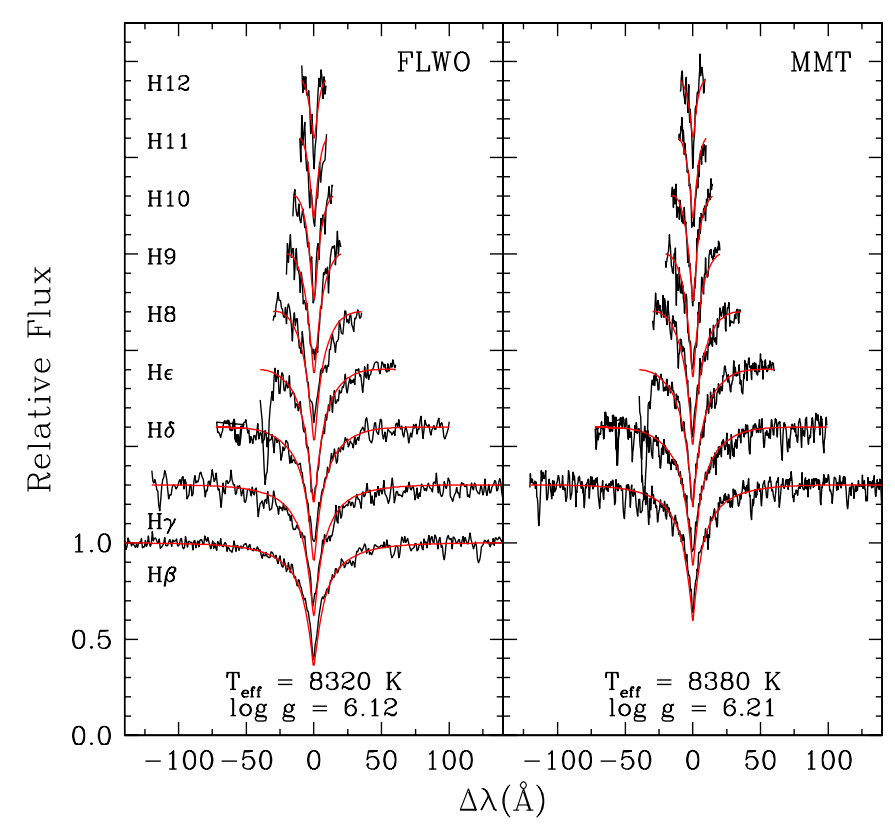

Figure 2. Model atmosphere fits (red) to the observed hydrogen Balmer lines (black) from $\mathrm{H} \beta$ to $\mathrm{H} 12$ for the spectrum taken at FLWO (left) and from $\mathrm{H} \gamma$ to $\mathrm{H} 12$ for the spectrum obtained at the MMT (right). The values of $T_{\text {eff }}$ and $\log g$ obtained from the individual fits are given at the bottom of each panel. The individual Balmer lines are normalized to unity and offset vertically by a factor of 0.3 for clarity. Note that we exclude the spectral range containing the Ca K line, from 3925 to $3945 \AA$ from both the normalization procedure and the fitting routine.

(A color version of this figure is available in the online journal.)

the same object. External uncertainties are typically $1.2 \%$ in $T_{\text {eff }}$ and 0.038 dex in $\log g$ (see Liebert et al. 2005 for details).

We present in Figure 2 our fits to the normalized Balmer lines for both the FLWO (left) and MMT (right) spectra. We exclude the wavelength region from $3925 \AA$ to $3945 \AA$, in the blue wing of $\mathrm{H} \epsilon$, from both our normalization and fitting procedures due to the presence of the strong Ca II K line at $3933 \AA$. The fits yield $T_{\text {eff }}=8320 \pm 120 \mathrm{~K}$ and $\log g=6.12 \pm 0.09$ for the FLWO spectrum and $T_{\text {eff }}=8380 \pm 120 \mathrm{~K}$ and $\log g=6.21 \pm 0.07$ for the MMT spectrum. The somewhat large uncertainties in $\log g$ are likely due to the presence of the many metal lines in the wings of the Balmer lines, which makes it difficult to accurately model the line widths, which are very sensitive to variations in $\log g$. Nonetheless, the results are consistent within the uncertainties, and we adopt the atmospheric parameters obtained from the MMT spectrum for the remainder of our analysis owing to the higher $\mathrm{S} / \mathrm{N}$ of those observations.

In order to determine a mass for J0745, we employ the new evolutionary calculations for ELM WDs presented in Althaus et al. (2013). In fact, we have employed a slightly finer grid of models than the ones published in the aforementioned article, kindly provided to us by L. Althaus (2013, private communication). These models predict a mass of $M_{1}=0.164 \pm$ $0.003 M_{\odot}$, as well as a cooling age of $\tau_{\text {cool }}=4.2 \pm 0.6$ Gyr. The uncertainty in mass (and age) is a combination of the uncertainties provided for each model in the grid with our uncertainties in $T_{\text {eff }}$ and $\log g$. However, we suspect that the model uncertainties are somewhat optimistic, and we adopt an uncertainty of $0.16 \pm 0.02 M_{\odot}(\sim 10 \%)$.

Given the mass, we then combine $\log g$ and $M_{1}$ to compute a radius of $R_{1}=0.053 \pm 0.008 R_{\odot}$. Next, by coupling our determinations of $T_{\text {eff }}, \log g$, and $R_{1}$ with the photometric 


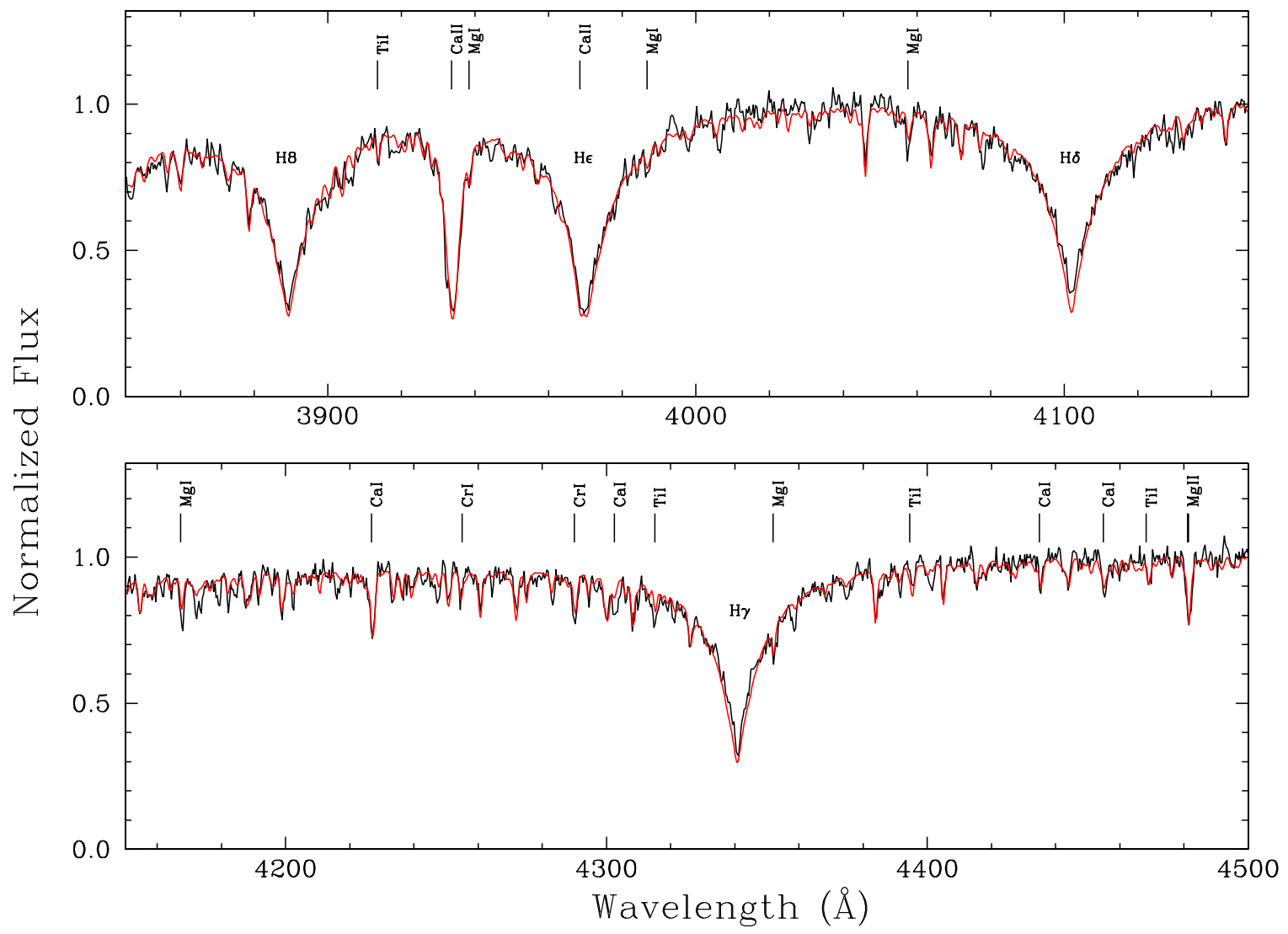

Figure 3. Our best-fit metal blanketed model (red) using $T_{\text {eff }}$ and $\log g$ from the Balmer line fits plotted over the observed MMT spectrum (black) of J0745. The Balmer lines from $\mathrm{H} \gamma$ to $\mathrm{H} 8$ are indicated in the figure, as well as lines of $\mathrm{Mg}$ I, Mg II, Ca I, Ca II, Ti I, and Cr I. All the remaining lines are Fe I lines.

(A color version of this figure is available in the online journal.)

calibrations of Holberg \& Bergeron (2006), we obtain an absolute magnitude of $M_{g}=9.8 \pm 0.4$. Finally, we are able to combine the absolute and apparent magnitudes of J0745 to calculate a distance of $d=201 \pm 36 \mathrm{pc}$. These derived quantities should be viewed with some caution considering the uncertainties in the evolutionary models on which they depend. Future observations and improved model calculations will permit more robust determinations. In particular, the distance to J0745 can be confirmed through ground- or space-based parallax observations such as will become available with the upcoming Gaia mission (Perryman et al. 2001).

\subsection{Metal Abundances}

Our method for fitting the multitude of metal lines in the spectrum of J0745 follows the procedure outlined in Dufour et al. (2012), which considers 15-30 ̊ wide segments centered on each of the strongest absorption features rather than fitting each individual line. We note that in many cases, more than one line from a given fitted element can be found in a single segment. In these cases, the measured abundance from that segment is essentially a weighted average of the lines, more weight (more frequency points) being attributed to the strongest lines. We finally take as the measured abundance of an element the average over all the segments.

We display in Figure 3 our best-fit solution for J0745, which includes $\mathrm{Mg}, \mathrm{Ca}, \mathrm{Ti}, \mathrm{Cr}$, and $\mathrm{Fe}$. We determine abundances, by number, of $\log (\mathrm{Mg} / \mathrm{H})=-3.90 \pm 0.30, \log (\mathrm{Ca} / \mathrm{H})=$ $-5.80 \pm 0.30, \log (\mathrm{Ti} / \mathrm{H})=-6.10 \pm 0.30, \log (\mathrm{Cr} / \mathrm{H})=$ $-5.60 \pm 0.30$, and $\log (\mathrm{Fe} / \mathrm{H})=-4.50 \pm 0.30$. The uncertain- ties on the abundances reflect the observed dispersion in the abundance measurements obtained from the individually fitted segments. The large dispersion can be attributed to a combination of the spectral resolution as well as the strength (near the noise level in many cases) and total number of observed metal lines. A high-S/N, high-resolution spectrum will permit more accurate determinations.

We see in Figure 3 that the observed line strengths are not all reproduced exactly, though the overall match with our spectroscopic solution is quite good, particularly for most of the strong $\mathrm{Ca}$ and $\mathrm{Mg}$ features in the spectrum. Moreover, we remark that the abundances for $\mathrm{Mg}, \mathrm{Ti}$, and $\mathrm{Cr}$ are super-solar, the $\mathrm{Ca}$ abundance is slightly sub-solar, and the $\mathrm{Fe}$ abundance is approximately solar when compared to the solar abundances listed in Asplund et al. (2005).

Finally, the fact that these abundances were determined from synthetic spectra calculated from a structure corresponding to an atmosphere of pure hydrogen could be considered as an additional source of uncertainty. It is true that in hot WDs, metals can significantly affect the thermodynamic structure of the atmosphere since ionized metals are important sources of opacity. However, J0745 is too cool for the metals to have a similar impact. In their analysis of GD 362 (which is $\sim 1500 \mathrm{~K}$ hotter than J0745), Gianninas et al. (2004) noted that synthetic spectra computed from a hydrogen pure model atmosphere and a metal-blanketed model were identical. However, our solution cannot be considered final since our analysis was done for fixed values of $T_{\text {eff }}$ and $\log g$ as measured from the Balmer line fits. The relative composition of the polluting elements, however, is much less sensitive to the exact final parameters adopted and can 

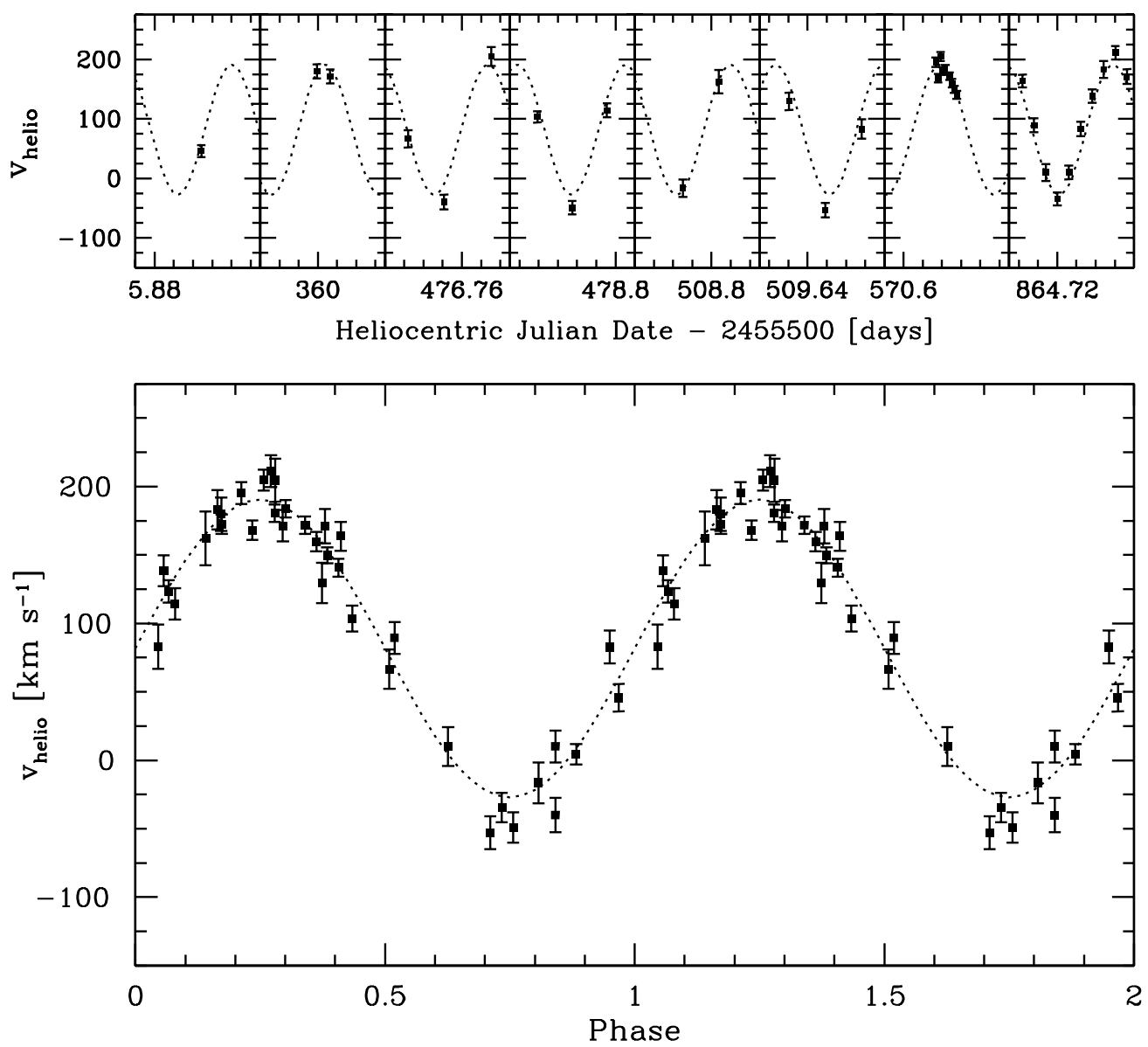

Figure 4. Radial velocities vs. time, plotted with a tick size of 0.02 days (upper panel), and phased to the best-fit spectroscopic orbital solution (lower panel).

thus be used as a high-precision measurement of the accreted material if radiative levitation is negligible (see Chayer 2013).

\subsection{Orbital Solution}

Figure 4 plots the radial velocities versus time (upper panel) and the radial velocities phased to the best-fit orbital solution (lower panel). We calculate orbital elements using the code of Kenyon \& Garcia (1986) as described in our earlier ELM Survey papers (Brown et al. 2013, and references therein). J0745 has a velocity semi-amplitude $K=108.7 \pm 2.9 \mathrm{~km} \mathrm{~s}^{-1}$, a systemic velocity $\gamma=81.7 \pm 4.3 \mathrm{~km} \mathrm{~s}^{-1}$, and an orbital period $P_{\text {spec }}=2.6975 \pm 0.2 \mathrm{hr}$. The uncertainty in radial velocity orbital period is large because there are period aliases near the best-fit value, at 2.7078, 2.6873, and $2.4326 \mathrm{hr}$. Based on these determinations, the resultant binary mass function is $f=0.015 \pm 0.002 M_{\odot}$.

For an edge-on orbit $\left(i=90^{\circ}\right)$, the mass function provides a lower limit for the companion mass, which in this case yields $M_{2}>0.102 \pm 0.014 M_{\odot}$. If we assume the mean inclination angle for a random stellar sample, $i=60^{\circ}$, we get an estimate of the most probable companion mass. For J0745, the most likely companion mass is $0.124 \pm 0.017 M_{\odot}$. This companion mass is still rather low, even by ELM WD standards, and so we suspect that this is a low-inclination system with an older, fainter, and likely more massive WD companion (see Section 4.5 for further discussion). Alternatively, assuming a random orbital inclination distribution allows us to calculate the probability of different companion masses. Specifically, there is a $92 \%$ probability that the companion is another low-mass WD with $M_{2} \leqslant 0.45 M_{\odot}$.
Finally, general relativity predicts that short-period binaries like J0745 lose orbital angular momentum due to gravitational wave radiation. The timescale for the binary to shrink and begin mass transfer via Roche-lobe overflow is $\tau_{\text {merger }} \leqslant 5.4$ Gyr. If we assume the most probable companion mass of $0.12 M_{\odot}$, J0745 will begin mass transfer in $<4.6$ Gyr. However, if J0745 really is a low-inclination system, then it will begin merging in only a few Gyr (e.g., $\tau_{\text {merger }} \sim 2$ Gyr for $i=30^{\circ}$ ).

\subsection{Light-curve Modeling}

Figure 5 shows the Argos light curve for J0745 (top panel) folded over the best-fit orbital period. The dashed red line corresponds to the best-fit orbital solution, as discussed in the following analysis. Additionally, we have computed a Fourier transform of our time-series photometry (Figure 5, bottom panel). With less than $7 \mathrm{hr}$ of data spread over five months, there is considerable aliasing around our highest peak, which occurs at $205.850 \pm 0.063 \mu \mathrm{Hz}$. Assuming that this signal is the result of ellipsoidal variations peaking twice per orbit, this corresponds to an orbital period of $P_{\text {orb }}=2.69883 \pm 0.00082 \mathrm{hr}$. We use this period to fold our time-series photometry in the top panel; this period agrees well with the orbit derived from the spectroscopy of $2.6975 \mathrm{hr}$.

There are five major effects that can cause photometric variability in a binary system: Doppler beaming, reprocessed light (reflection), ellipsoidal variations, eclipses, and pulsations. To best characterize the variability in the light curve, we perform a Monte Carlo analysis by creating $10^{5}$ synthetic light curves in which we replace the measured flux $f$ with $f+g \delta f$, where $\delta f$ is the error in flux and $g$ is a Gaussian deviate with zero mean 

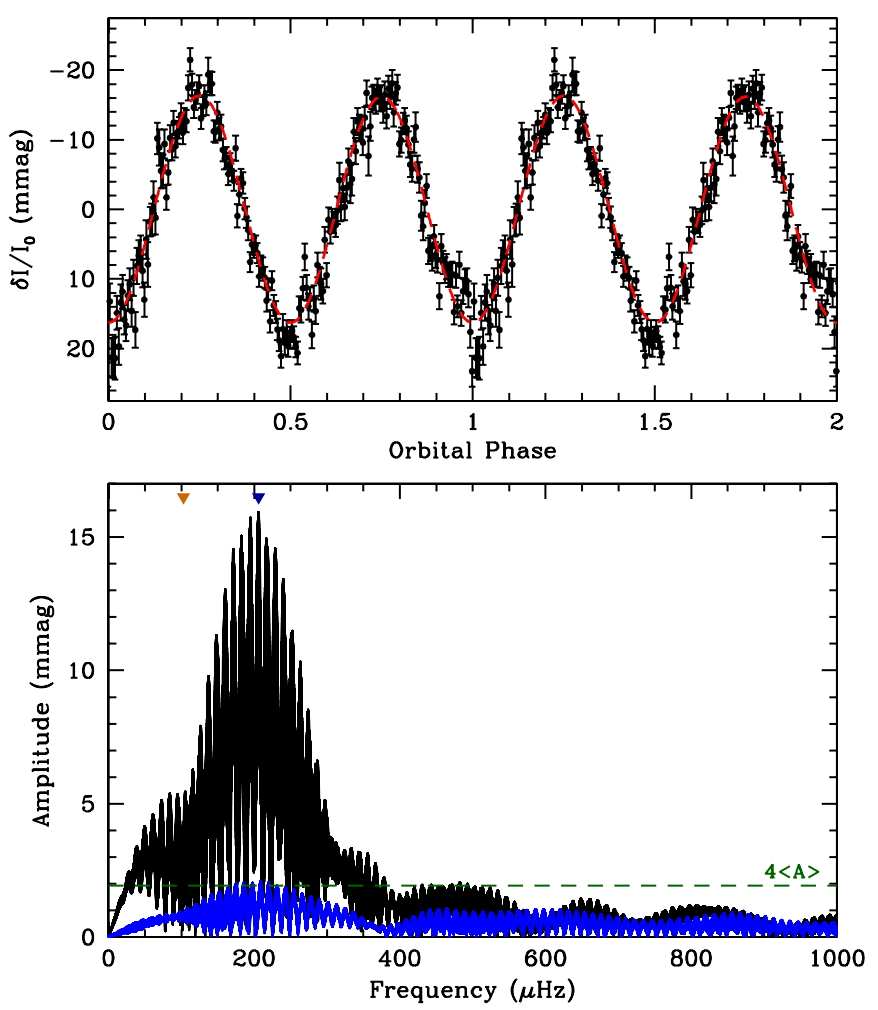

Figure 5. Time-series photometry of J0745+1949. The top panel shows the optical light curve, folded at the orbital period and repeated for clarity. The dashed red line represents the best-fit orbital solution. The bottom panel shows a Fourier transform of the target (black) and brightest comparison star (blue). The orange and dark blue triangles mark the orbital period and half-orbital period, respectively, as determined from the time-series spectroscopy. Variability in the comparison star shows evidence of atmospheric variability at similar timescales as the period of interest in the target, which may explain why the photometry does not phase exceptionally well. The spectroscopy and photometry both find $\sim 2.7 \mathrm{hr}$ as the best-fit orbital period.

(A color version of this figure is available in the online journal.)

and unit variance (e.g., Hermes et al. 2012a). We then fit each light curve with a five-parameter model that includes an offset, and the sine and cosine terms that represent Doppler beaming $(\sin \phi)$, ellipsoidal variations $(\cos 2 \phi)$, reflection $(\cos \phi)$, and the first harmonic of the orbital period $(\sin 2 \phi)$.

We detect a high-amplitude signal consistent with ellipsoidal variations of the low-mass, visible primary; this is manifest as a $\cos 2 \phi$ modulation in the light curve. Our Monte Carlo analysis yields an amplitude of $\cos 2 \phi=1.54 \% \pm 0.12 \%$ for the ellipsoidal variations; no other (co)sine terms appear with any statistical significance. Although we expect a $0.15 \%$ Doppler beaming signal given $K_{1}$, the temperature of the primary, and our blue-bandpass filter (Shporer et al. 2010), we do not detect this low-amplitude $\sin \phi$ modulation at the orbital period with any significance.

Tidal distortions do not cause a perfectly ellipsoidal shape, so we represent the ellipsoidal variations as harmonics to the first four $\cos \phi$ terms as derived in Morris \& Naftilan (1993). Equation (1) in that work theoretically predicts the ellipsoidal variation amplitude, which is dominated by

$$
L(\phi) / L_{0}=\frac{-3\left(15-u_{1}\right)\left(1+\tau_{1}\right)\left(R_{1} / a\right)^{3} q \sin ^{2} i}{20\left(3-u_{1}\right)} \cos 2 \phi
$$

where $q=M_{2} / M_{1}$ is the mass ratio, $u_{1}$ and $\tau_{1}$ are the linear limb-darkening and gravity-darkening coefficients for the primary, respectively, $a$ is the orbital semi-major axis of the system, $i$ is the system inclination and $R_{1}$ is the radius of the primary. Here we will assume $u_{1}=0.58$ for this relatively cool ELM WD, as calculated by Gianninas et al. (2013).

We use the $1.54 \% \pm 0.12 \%$ amplitude ellipsoidal variations to infer the radius of the ELM WD primary as a function of the inclination angle, since all unknown parameters in Equation (1) can be written in terms of $i$. This result is shown in Figure 6. We assume that energy transport at the surface is primarily radiative and thus the gravity darkening coefficient is $\tau_{1}=1.0$, but we have also considered $\tau_{1}=0.36$ for the non-radiative case in our $1 \sigma$ uncertainties shown in Figure 6.

The radius inferred from the ellipsoidal variations of the primary appears considerably larger than we would expect given its surface gravity and mass; we expect a radius of $0.053 R_{\odot}$ from the Althaus et al. (2013) models, but divergently we observe a minimum radius at high inclinations of more than $0.13 R_{\odot}$. We caution that system parameters derived from the ellipsoidal variation amplitude using the approximation of Morris \& Naftilan (1993) are only valid when the primary is corotating with the binary orbit, as was demonstrated for KOI-74 by Bloemen et al. (2012) and may not be the case here. We discuss this discrepancy further in Section 5.2.

The low $\log g$ and $T_{\text {eff }}(8380 \mathrm{~K})$ of $\mathrm{J} 0745$ put it near the instability strip for pulsations in ELM WDs (Hermes et al. 2013). However, we see no evidence for variability at timescales other than the orbital- and half-orbital periods, to a limit of $0.4 \%$ in relative amplitude.

\subsection{Spectral Energy Distribution}

The available UV-optical-IR photometry for J0745 is listed in Table 1. Since J0745 is situated at a galactic latitude of $b \approx 20^{\circ}$, interstellar extinction must be taken into consideration. Consequently, we have applied corrections to both the GALEX and SDSS photometry. For the GALEX bands we have adopted extinction coefficients of $A_{\mathrm{FUV}} / E(B-V)=8.24$ and $A_{\mathrm{NUV}} /(E(B-V)=8.2$, as prescribed by Wyder et al. (2007), and combined these with a reddening of $E(B-V)=$ 0.0614 as provided for the coordinates of $\mathrm{J} 0745.6$ In addition, we adopt the extinction corrections for each SDSS band as provided on the SDSS Skyserver. ${ }^{7}$ Both the GALEX and SDSS extinction corrections are based on the dust maps of Schlegel et al. (1998). The corrected photometry is displayed in Figure 7 as the colored error bars. We plot as well the full spectral energy distribution (SED) for a model corresponding to our adopted spectroscopic solution of $T_{\text {eff }}=8380 \mathrm{~K}$ and $\log g=$ 6.21. Furthermore, we have scaled the flux of the SED using our determinations of the radius and distance (see Table 2) as the observed flux, $f_{\lambda, m}$, and the Eddington flux, $H_{\lambda, m}$, are related by,

$$
f_{\lambda, m}=4 \pi(R / d)^{2} H_{\lambda, m}
$$

where $R / d$ is the ratio of the radius of the star to its distance from Earth. The agreement between the SED of our spectroscopic solution and the observed photometry is quite good overall. We do note a significant difference in the two sets of SDSS photometry, particularly in the $r$-band measurements. Some of

\footnotetext{
6 http://galex.stsci.edu/GR6/?page=explore $\&$ photo $=$ true $\&$ objid $=$ 6407287600137114823

7 http://skyserver.sdss3.org/dr9/en/tools/explore/obj.asp?ra=116.29817\& $\mathrm{dec}=19.82403$
} 


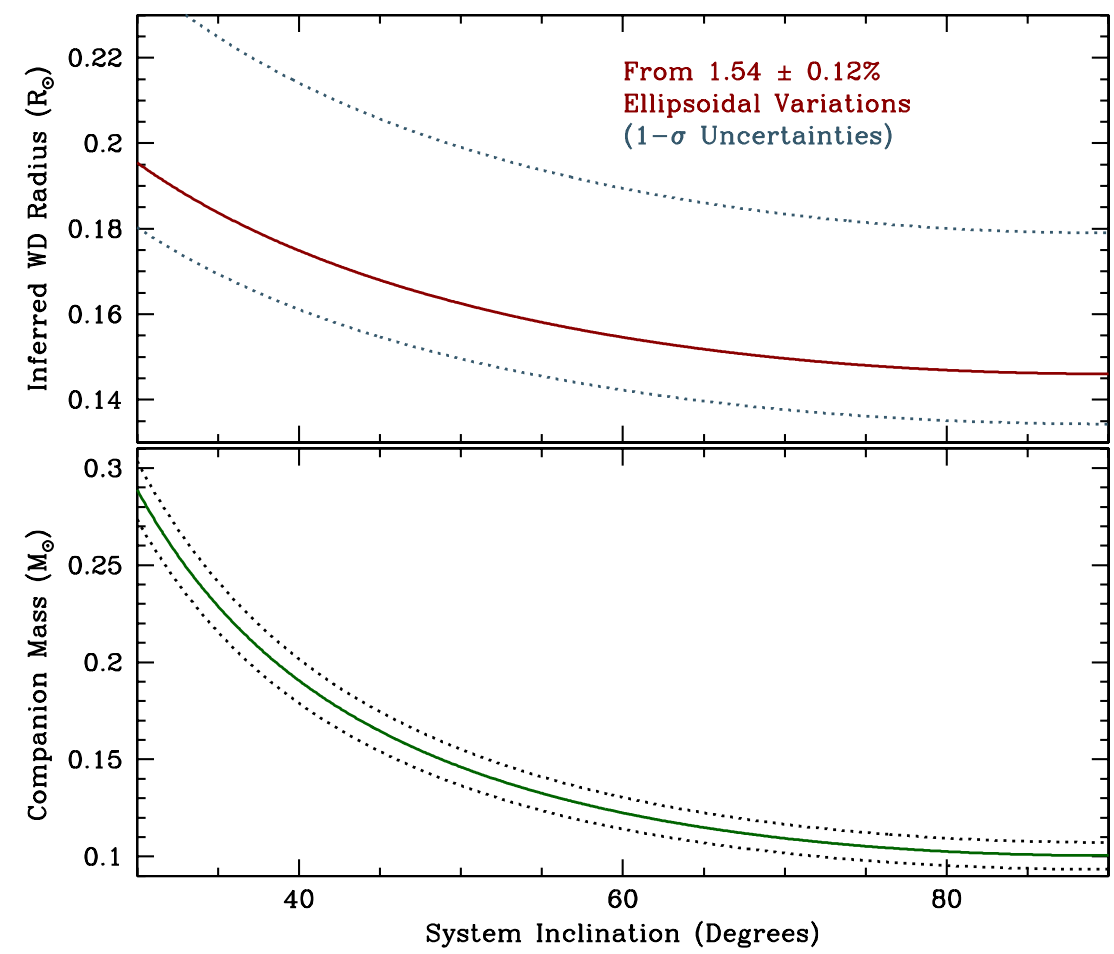

Figure 6. Top panel shows allowed values for the radius, modulo the unknown system inclination, to explain the $1.54 \% \pm 0.12 \%$ amplitude ellipsoidal variations observed in J0745+1949, and includes $1 \sigma$ uncertainties. The larger upper limit is a result of differing the gravity darkening coefficient. If we use the determined surface gravity and mass to predict an expected ELM WD radius, we do not expect an ELM WD with a radius larger than $0.06 R_{\odot}$, which is much smaller than the lower limit we measure from the ellipsoidal variations. The bottom panel shows the corresponding companion mass from the spectroscopic mass function, including $1 \sigma$ uncertainties from the derived primary mass.

(A color version of this figure is available in the online journal.)

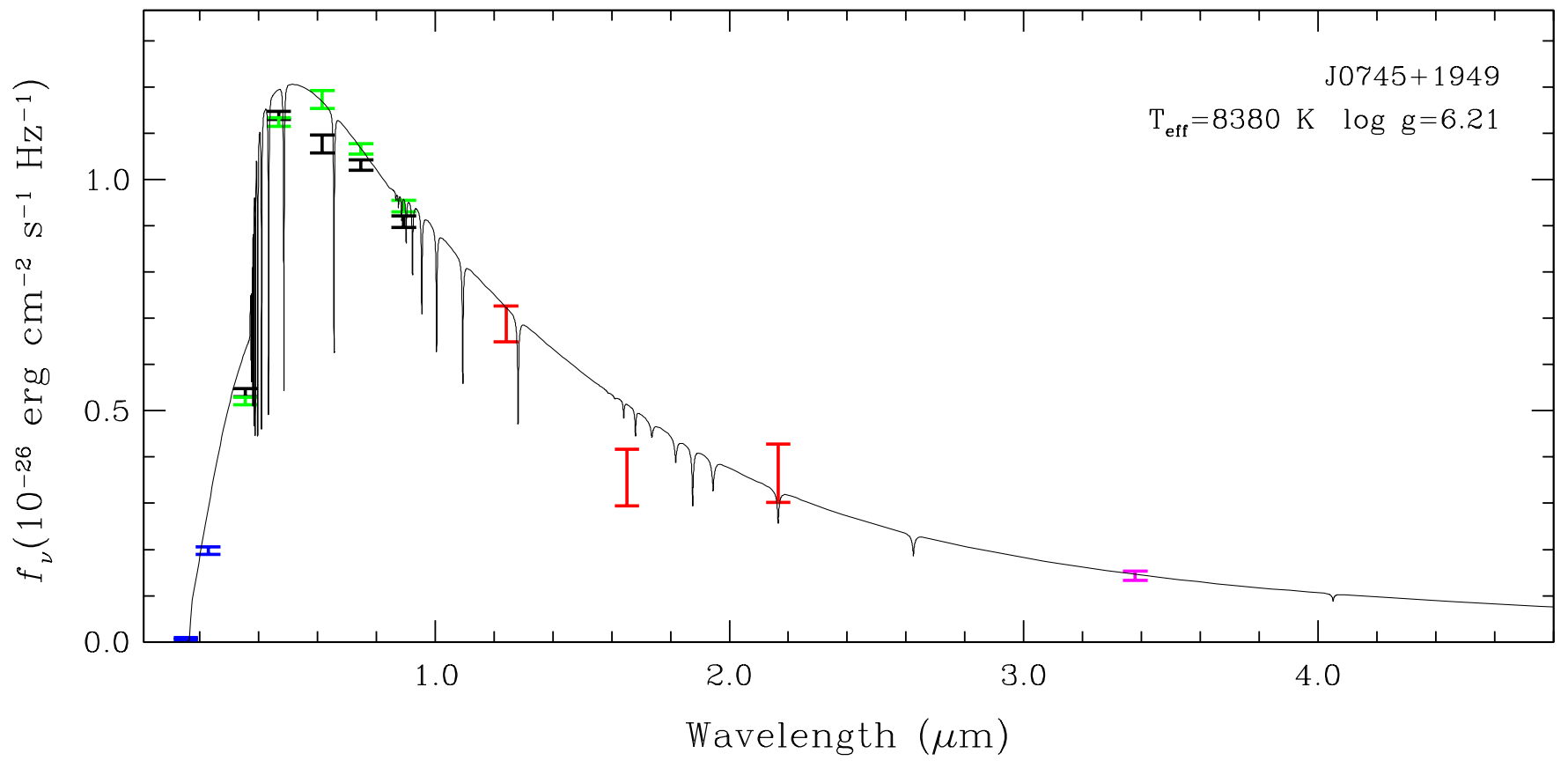

Figure 7. Plot of the combined GALEX (blue), SDSS (2013 January, black; 2013 February, green), 2MASS (red), and WISE (magenta) photometry. The thin black line represents the spectral energy distribution for our best-fit spectroscopic solution scaled to the observed photometry by our radius and distance determinations (see Equation (2)).

(A color version of this figure is available in the online journal.)

this variation can be attributed to the observed photometric variability of J0745 due to ellipsoidal variations. Furthermore, we do not see any features that would signal the presence of the companion. If we assume that the companion is indeed a more massive and cooler WD, then it is necessarily much fainter. Finally, there is no evidence of any IR excess from a potential debris disk from which J0745 would actively be accreting the metals observed in its optical spectrum. 


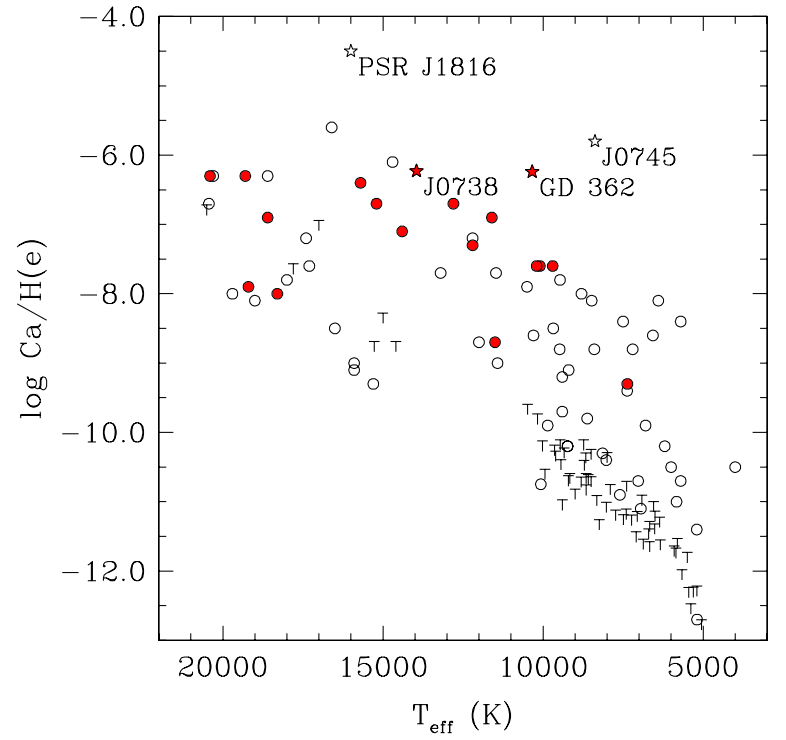

Figure 8. Published Ca abundances (white circles), or upper limits (crosses), for 133 polluted WDs given with respect to the main atmospheric constituent (i.e., $\log (\mathrm{Ca} / \mathrm{H})$ or $\log (\mathrm{Ca} / \mathrm{He}))$, as a function of $T_{\text {eff }}$. Abundance determinations are taken from Zuckerman et al. (2003, 2007), Berger et al. (2005), Koester et al. (2005), Farihi et al. (2009, 2010b), Dufour et al. (2012), and Kaplan et al. (2013). Stars denote objects of particular interest that are discussed in the text. Finally, filled red symbols correspond to WDs with known debris disks.

(A color version of this figure is available in the online journal.)

Table 2

System Properties for J0745

\begin{tabular}{lc}
\hline \hline Parameter & Value \\
\hline$T_{\text {eff }}(\mathrm{K})$ & $8380 \pm 120$ \\
$\log g$ & $6.21 \pm 0.07$ \\
$\log (\mathrm{Mg} / \mathrm{H})$ & $-3.90 \pm 0.30$ \\
$\log (\mathrm{Ca} / \mathrm{H})$ & $-5.80 \pm 0.30$ \\
$\log (\mathrm{Ti} / \mathrm{H})$ & $-6.10 \pm 0.30$ \\
$\log (\mathrm{Cr} / \mathrm{H})$ & $-5.60 \pm 0.30$ \\
$\log (\mathrm{Fe} / \mathrm{H})$ & $-4.50 \pm 0.30$ \\
$P_{\text {spec }}(\mathrm{hr})$ & $2.6975 \pm 0.02$ \\
$P_{\text {phot }}(\mathrm{hr})$ & $2.6988 \pm 0.0008$ \\
$\left.K(\mathrm{~km} \mathrm{~s})^{-1}\right)$ & $108.7 \pm 2.9$ \\
$\gamma\left(\mathrm{km} \mathrm{s}{ }^{-1}\right)$ & $81.7 \pm 4.3$ \\
$M_{g}$ & $9.75 \pm 0.39$ \\
$d(\mathrm{pc})$ & $201 \pm 36$ \\
$M_{1}\left(M_{\odot}\right)$ & $0.16 \pm 0.02$ \\
$R_{1, \text { spec }}\left(R_{\odot}\right)$ & $0.053 \pm 0.008$ \\
$\tau_{\text {cool }}(\mathrm{Gyr})$ & $4.232 \pm 0.594$ \\
Mass function $\left(M_{\odot}\right)$ & $0.015 \pm 0.002$ \\
$M_{2}\left(M_{\odot}\right)$ & $>0.102 \pm 0.014$ \\
$M_{2, i=60^{\circ}}\left(M_{\odot}\right)$ & $0.124 \pm 0.017$ \\
$\tau_{\text {merger }}(\mathrm{Gyr})$ & $<5.447 \pm 1.374$ \\
$a(\mathrm{AU})$ & $>0.0029 \pm 0.0003$ \\
$a\left(R_{\odot}\right)$ & $>0.630 \pm 0.058$ \\
\hline
\end{tabular}

\section{DISCUSSION}

\subsection{Origin of Metals}

J0745 is truly a unique system that allows us to study several phenomena simultaneously. First and foremost are the very high metal abundances we observe. Indeed, J0745 stands out when compared to other metal polluted WDs. In Figure 8 we plot published $\mathrm{Ca}$ abundances (or upper limits, see figure caption) for metal-polluted WDs as a function of $T_{\text {eff }}$. The WD companion of PSR J1816+4510 (Kaplan et al. 2013) is also shown and currently has the highest $\mathrm{Ca}$ abundance measured

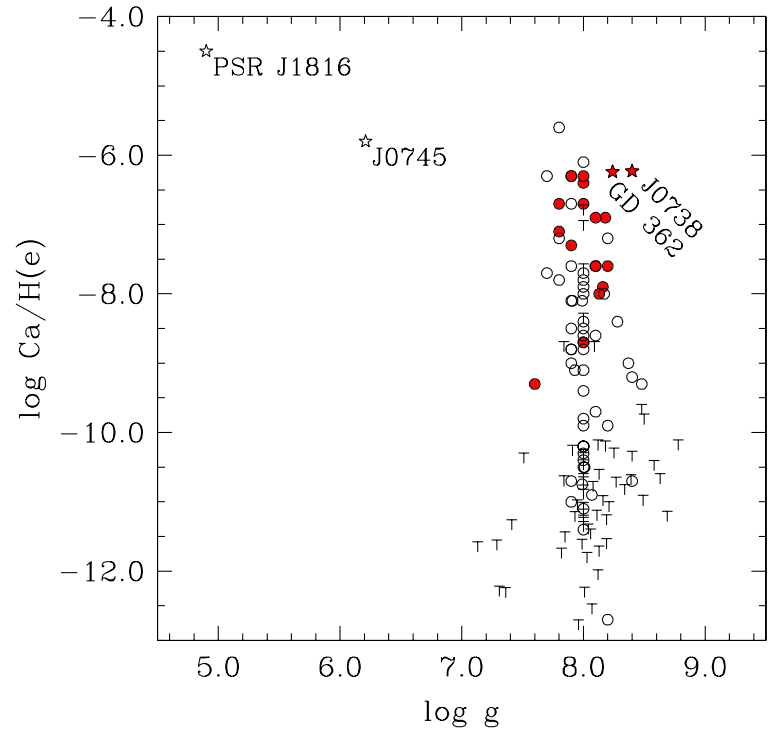

Figure 9. Same as Figure 8 but with the $\mathrm{Ca}$ abundances plotted as a function of $\log g$ instead.

(A color version of this figure is available in the online journal.)

for a WD. Figure 8 also includes the extremely metal-rich DBZ, J0738+1835 (Dufour et al. 2012), and GD 362 (Zuckerman et al. 2007). J0745 clearly stands apart from the majority of the other WDs in Figure 8 and has the highest $\mathrm{Ca}$ abundance when compared to WDs with similar $T_{\text {eff }}$. Similarly, Figure 9 shows the same $\mathrm{Ca}$ abundances plotted as a function of $\log g$. Most objects cluster around $\log g \sim 8.0$, while GD 362 and J0738 are somewhat more massive. On the other hand, J0745 and the WD companion to PSR J1816 have $\log g$ roughly two and three orders of magnitude lower, respectively, highlighting once again the extreme nature of these objects. Naturally, this leads us to inquire about the source of these metals.

The currently accepted paradigm for explaining the presence of metals in more massive cool WDs is accretion from a circumstellar debris disk. Indeed, we see in Figure 8 that debris disks around many of the polluted WDs have been detected. However, we have seen that there is no readily observable IR excess in the available IR photometry of J0745. Debris disks around more massive WDs tend to be found within $\sim 0.1-1.0 R_{\odot}$, the typical tidal disruption radius for WDs (von Hippel et al. 2007; Farihi et al. 2010b). However, we expect that in the case of ELM WD binaries these debris disks will be circumbinary and not simply circumstellar. Combining our determinations for the mass of the primary, $M_{1}$, the minimum companion mass, $M_{2}$, and the orbital period, $P_{\text {spec }}$, we compute a minimum orbital separation of $a>0.0029 \pm 0.0003 \mathrm{AU}$ or equivalently $a>0.63 \pm 0.06 R_{\odot}$. In order to explore whether a debris disk can explain the observed heavy elements, we have computed a series of debris disk models following the formalism of Jura (2003). We note that our models assume an optically thick disk. The orbital stability calculations of Holman $\&$ Wiegert (1999) show that for a circularized binary orbit (i.e., $e=0$ ) the critical radius for a system with a mass ratio $>0.3$ is $\sim 2$ times the orbital separation. Presuming the debris disk to be circumbinary, we chose an inner radius of $1.3 R_{\odot}$ and outer radii of $1.4,1.5,2.0,3.0$ and $5.0 R_{\odot}$. We plot the result of our debris disk calculations in Figure 10. If a debris disk corresponding to the parameters we assumed for our models were present, it would likely go undetected. Only the widest and least inclined disks would be detected in the $W 1$ band, given the uncertainties. 


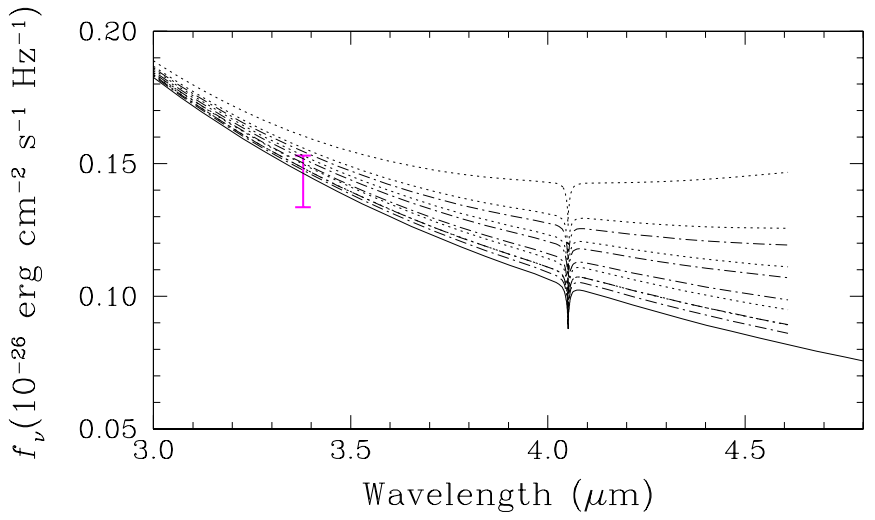

Figure 10. Similar to Figure 7 but showing only the wavelength region with $\lambda>3.0 \mu \mathrm{m}$. The dotted lines represent the combined WD and disk flux for disk models with outer disk radii of $R_{\text {out }}=1.4,1.5,2.0,3.0,4.0,5.0 R_{\odot}$, from bottom to top, respectively, and for an inclination angle of $i=30^{\circ}$. The dash-dotted lines represent equivalent WD and model disk model fluxes but assume, instead, an inclination angle of $i=60^{\circ}$. All the disk models assume an inner disk radius of $R_{\text {in }}=1.3 R_{\odot}$.

(A color version of this figure is available in the online journal.)

If J0745 does in fact harbor a debris disk, it would have to be in the form of a narrow ring or with an inclination angle high enough to limit the visible emission from the disk. The WISE data dictate that the disk could have an inclination of $i \geqslant 30^{\circ}$ with $R_{\text {out }} \leqslant 3.0 R_{\odot}$. This would allow it to escape detection through IR excess. Consequently, a debris disk scenario for J0745 cannot be entirely ruled out.

To further explore the possibility that J0745 has accreted its metals from a debris disk like other heavily polluted WDs, we compare the abundance ratios of the detected metals with those seen in J0738 and GD 362. Since we have not identified any $\mathrm{Si}$ in $\mathrm{J} 0745$, we use $\mathrm{Mg}$ as the reference element and compute abundance ratios relative to $\mathrm{Mg}$. The results of this exercise are displayed in Figure 11. We also plot the abundance ratios for solar and chondritic material based on the abundances in Lodders (2003). Unfortunately, the relatively small number of metals detected in J0745 with our medium-resolution MMT spectrum and the uncertainties in our abundance measurements preclude any meaningful conclusions regarding the origin of metals in J0745. Indeed, Figure 11 shows that all the abundance ratios are generally similar, with $\mathrm{Ca}$ and $\mathrm{Fe}$ being somewhat enhanced in GD 362, while Ti is slightly deficient in J0738. We can also obtain a rough estimate of the total mass of metals present in J0745. Assuming a mass of $M_{C Z} \approx 5 \times 10^{-9} M_{\odot}$ for the hydrogen convection zone (L. Althaus 2013, private communication) coupled with the metal abundances listed in Table 2, we estimate the total mass of metals in the photosphere of J0745 to be $M_{Z} \gtrsim 5 \times 10^{22} \mathrm{~g}$. This is roughly $1 / 20$ the mass of the dwarf planet Ceres, the largest asteroid in the solar system. This is an order of magnitude less than the mass of metals found in J0738 (Dufour et al. 2012). Our estimate necessarily represents a lower limit since we have not yet identified all the metals present in the atmosphere of J0745.

Another factor to consider is the diffusion timescale, $\tau_{D}$, for metals in the atmosphere of an ELM WD. In more massive WDs, the diffusion timescale is usually several orders of magnitude shorter than the WD cooling age, $\tau_{\text {cool }}$. This is one of the reasons that accretion must necessarily be ongoing as any material from a previous accretion episode would have long ago settled out of the atmosphere. On the other hand, the much weaker surface gravity of ELM WDs will likely allow heavy elements to diffuse

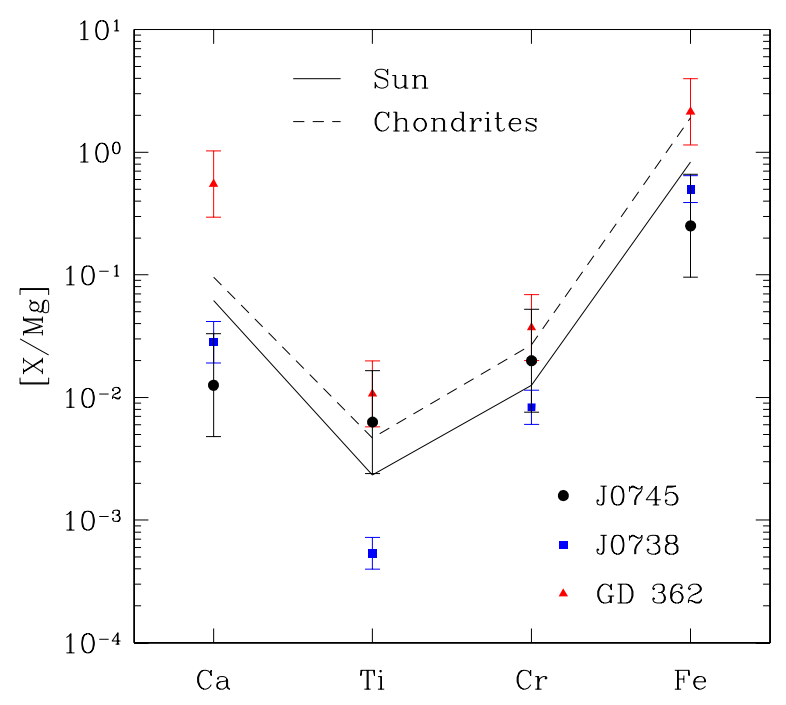

Figure 11. Elemental abundances by number relative to magnesium. The GD 362 data are from Zuckerman et al. (2007), the J0738 data are taken from Dufour et al. (2012), and the solar and chondritic abundances are from Lodders (2003).

(A color version of this figure is available in the online journal.)

more slowly. Koester \& Wilken (2006) compute diffusion times for normal WDs, but not for the ELM WD regime. If we consider the models with $T_{\text {eff }}=8000 \mathrm{~K}$, we notice that the logarithm of the diffusion timescale for $\mathrm{Ca}, \mathrm{Mg}$, and $\mathrm{Fe}$ varies roughly linearly as a function of $\log g$. Specifically, $\log \tau_{D}$ increases by $\sim 0.5$ dex for every decrease of $0.25 \mathrm{dex}$ in $\log g$ for $\mathrm{Mg}$, and $\mathrm{Fe}$, and by $\sim 0.4 \mathrm{dex}$ for $\mathrm{Ca}$. If we follow this trend, we can extrapolate that for $\log g \sim 6.25$, the values of $\log \tau_{D}$ for $\mathrm{Ca}$, $\mathrm{Mg}$, and $\mathrm{Fe}$ are $\sim 5.6, \sim 6.7$, and $\sim 6.6$, respectively. The models of Althaus et al. (2013) predict that the cooling age for J0745 is $\log \tau_{\text {cool }} \approx 9.6$. We see that even in ELM WDs, the diffusion timescale is still much shorter than the evolutionary timescale despite the weaker gravitational field. Note that the calculations of Koester \& Wilken (2006) assume a steady-state accretion scenario, which may or may not be the case here. The accretion rate depends on both the mass of the WD and the diffusion timescale. Both of these quantities are not well constrained. Consequently, any calculation of the accretion rate at this time would be speculative, and we leave such a determination for a future publication.

Yet another mechanism that might be at work in J0745 is radiative levitation. It is well established that heavy elements can be maintained in the atmospheres of very hot WDs by radiative forces (see, e.g., Chayer et al. 1995a, 1995b). Recent calculations by Chayer \& Dupuis (2010) demonstrate that WDs with $\log g=8.0$ and as cool as $T_{\text {eff }}=20,000 \mathrm{~K}$ can potentially sustain C, Al, and $\mathrm{Si}$ in their atmospheres. J0745's surface gravity is two orders of magnitude weaker and its temperature is $12,000 \mathrm{~K}$ cooler than the models presented in Chayer \& Dupuis (2010). Consequently, the radiation field might not be intense enough, even at $\log g \sim 6.0$, to overcome the local gravitational force. However, radiative levitation calculations for the appropriate regime of $T_{\text {eff }}$ and $\log g$ are needed to conclusively show if heavy elements can be sustained in the atmospheres of ELM WDs by this mechanism.

It is also possible that a recent shell flash could have mixed up the interior of the star bringing metals to the surface. A recent shell flash is a plausible explanation for the WD companion to PSR J1816+4510 (Kaplan et al. 2013) which has $T_{\text {eff }}=$ $16,500 \mathrm{~K}, \log g=4.90$, and a cooling age of $\approx 85 \mathrm{Myr}$ (Althaus 
et al. 2013). A recent shell flash seems unlikely for J0745 given its mass $\left(0.16 M_{\odot}\right)$ and cooling age $(4.2 \mathrm{Gyr})$. However, there is still much uncertainty in ELM WD evolutionary models despite the recent improvements presented in Althaus et al. (2013) when compared to previously available calculations (e.g., Panei et al. 2007). It would therefore be premature to discount the shell flash scenario at this point in time based solely on our determination of the cooling age.

\subsection{Surface Gravity}

There is a discrepancy between the stellar radius measured from the ellipsoidal variations and the stellar radius measured from our $\log g$ coupled with the Althaus et al. (2013) models. This is not the first instance where we have encountered an issue in reconciling the radius of an ELM WD, estimated through other means, with our spectroscopic measurement of $\log g$. Indeed, the runaway binary LP 400-22 poses an equally baffling mystery since its $\log g$ determined from Balmer line fitting is at odds with the $\log g$ inferred from parallax measurements (for a detailed discussion, see Kilic et al. 2013b). As a result, we must consider another source of uncertainty. For normal mass WDs, a long-standing issue has been that of the "high $\log g$ problem" for WDs with $T_{\text {eff }}<13,000 \mathrm{~K}$ (Kepler et al. 2007; Tremblay et al. 2010; Gianninas et al. 2011). Over the years, various causes were proposed and explored. The root of the problem was finally exposed by Tremblay et al. (2011), who showed that the 1D treatment of convection using the mixinglength theory (MLT; Böhm-Vitense 1958; Bohm \& Cassinelli 1971) was the true culprit. Tremblay et al. (2013a) presented new models that employ a complete 3D hydrodynamical treatment of convection and demonstrated that the new models effectively eliminate the so-called high $\log g$ problem. If we consider that the atmosphere models we rely on for our analysis use MLT to model convection, we should logically expect them to suffer from the same high $\log g$ problem as well, albeit in a different regime of surface gravity. Recent calculations show that for $\log g=7.0$, the 3D models predict a surface gravity of $\sim 0.3 \mathrm{dex}$ lower than the 1D models (Tremblay et al. 2013b). Clearly, a grid of 3D models appropriate for ELM WDs is needed to explore these effects further and to resolve the discrepancy between the radius estimate from the photometric and spectroscopic data.

\subsection{Future Evolution}

J0745 has a merger time less than a Hubble time (see Table 2); it is of interest to explore what the ultimate fate of this system will be. The mass ratio of the system is $q>0.36$. Binary mass transfer models suggest that a system with this mass ratio will undergo unstable mass transfer and merge to form a single WD (Marsh et al. 2004).

\section{CONCLUSIONS}

We present the discovery of the most recent addition to the ELM WD family, J0745. Spectroscopic and photometric analyses demonstrate that J0745 is a cool, tidally distorted, compact ELM WD binary whose primary is polluted with nearly solar abundances of $\mathrm{Ca}, \mathrm{Mg}, \mathrm{Ti}, \mathrm{Cr}$, and $\mathrm{Fe}$. There is no evidence of IR excess from a debris disk from which J0745 could be accreting heavy elements, as is often observed in more massive WDs with atmospheric metal pollution. However, we cannot completely rule out the debris disk scenario since the geometry of the disk (inclination angle, radial extent) is unknown and could preclude its detection at $2-5 \mu \mathrm{m}$. On the other hand, radiative levitation, which would be favored in an ELM WD with its much weaker gravitational field, may also play a role in keeping metals in the atmosphere. Unfortunately, calculations for the radiative support of heavy elements in the atmospheres of ELM WDs are not yet available. Further progress requires a more accurate determination of the metal abundances. The UV spectrum of J0745, accessible with the Hubble Space Telescope's Cosmic Origins Spectrograph, is particularly useful for discriminating between the accretion, radiative levitation, and shell flash scenarios.

We wish to thank the referee for a careful reading of this manuscript and for useful suggestions that helped to improve it. We also thank P. Canton for useful discussions. J.J.H. and S.T.H. acknowledge the support of the NSF under grant AST-0909107 and the Norman Hackerman Advanced Research Program under grant 003658-0252-2009. This research makes use of the SAO/NASA Astrophysics Data System Bibliographic Service. This project makes use of data products from the Sloan Digital Sky Survey, which has been funded by the Alfred P. Sloan Foundation, the Participating Institutions, the National Science Foundation, and the U.S. Department of Energy Office of Science. M.K. acknowledges support from the NSF under grant AST-1312678. This work was supported in part by the Smithsonian Institution. This work is funded in part by the NSERC Canada.

Facilities: MMT (Blue Channel Spectrograph), FLWO:1.5 m (FAST), Struve (Argos)

\section{REFERENCES}

Ahn, C. P., Alexandroff, R., Allende Prieto, C., et al. 2012, ApJS, 203, 21

Althaus, L. G., Miller Bertolami, M. M., \& Córsico, A. H. 2013, A\&A, 557, A19

Asplund, M., Grevesse, N., \& Sauval, A. J. 2005, in ASP Conf. Ser. 336, Cosmic Abundances as Records of Stellar Evolution and Nucleosynthesis, ed. T. G. Barnes, III \& F. N. Bash (San Francisco, CA: ASP), 25

Barber, S. D., Patterson, A. J., Kilic, M., et al. 2012, ApJ, 760, 26

Berger, L., Koester, D., Napiwotzki, R., Reid, I. N., \& Zuckerman, B. 2005, A\&A, 444, 565

Bildsten, L., Shen, K. J., Weinberg, N. N., \& Nelemans, G. 2007, ApJL, 662, L95

Bloemen, S., Marsh, T. R., Degroote, P., et al. 2012, MNRAS, 422, 2600

Bohm, K. H., \& Cassinelli, J. 1971, A\&A, 12, 21

Böhm-Vitense, E. 1958, ZAp, 46, 108

Brown, W. R., Kilic, M., Allende Prieto, C., Gianninas, A., \& Kenyon, S. J. 2013, ApJ, 769, 66

Brown, W. R., Kilic, M., Allende Prieto, C., \& Kenyon, S. J. 2010, ApJ, 723, 1072

Brown, W. R., Kilic, M., Allende Prieto, C., \& Kenyon, S. J. 2012, ApJ, 744,142

Chayer, P. 2013, arXiv:1310.6245

Chayer, P., \& Dupuis, J. 2010, in AIP Conf. Proc. 1273, 17th European White Dwarf Workshop, ed. K. Werner \& T. Rauch (Melville, NY: AIP), 394 Chayer, P., Fontaine, G., \& Wesemael, F. 1995a, ApJS, 99, 189

Chayer, P., Vennes, S., Pradhan, A. K., et al. 1995b, ApJ, 454, 429

Debes, J. H., Hoard, D. W., Farihi, J., et al. 2012, ApJ, 759, 37

Debes, J. H., \& Sigurdsson, S. 2002, ApJ, 572, 556

Dufour, P., Kilic, M., Fontaine, G., et al. 2010, ApJ, 719, 803

Dufour, P., Kilic, M., Fontaine, G., et al. 2012, ApJ, 749, 6

Fabricant, D., Cheimets, P., Caldwell, N., \& Geary, J. 1998, PASP, 110, 79

Farihi, J. 2011, in AIP Conf. Proc. 1331, Planetary Systems beyond the Main Sequence, ed. S. Schuh, H. Drechsel, \& U. Heber (Melville, NY: AIP), 193

Farihi, J., Barstow, M. A., Redfield, S., Dufour, P., \& Hambly, N. C. 2010a, MNRAS, 404, 2123

Farihi, J., Jura, M., Lee, J.-E., \& Zuckerman, B. 2010b, ApJ, 714, 1386

Farihi, J., Jura, M., \& Zuckerman, B. 2009, ApJ, 694, 805

Gianninas, A., Bergeron, P., \& Ruiz, M. T. 2011, ApJ, 743, 138

Gianninas, A., Dufour, P., \& Bergeron, P. 2004, ApJL, 617, L57

Gianninas, A., Strickland, B. D., Kilic, M., \& Bergeron, P. 2013, ApJ, 766, 3 
Hermes, J. J., Kilic, M., Brown, W. R., Montgomery, M. H., \& Winget, D. E. 2012a, ApJ, 749, 42

Hermes, J. J., Kilic, M., Brown, W. R., et al. 2012b, ApJL, 757, L21

Hermes, J. J., Montgomery, M. H., Winget, D. E., et al. 2013, ApJ, 765, 102

Hoard, D. W., Debes, J. H., Wachter, S., Leisawitz, D. T., \& Cohen, M. 2013, ApJ, 770, 21

Holberg, J. B., \& Bergeron, P. 2006, AJ, 132, 1221

Holman, M. J., \& Wiegert, P. A. 1999, AJ, 117, 621

Hummer, D. G., \& Mihalas, D. 1988, ApJ, 331, 794

Jura, M. 2003, ApJL, 584, L91

Jura, M. 2006, ApJ, 653, 613

Jura, M. 2008, AJ, 135, 1785

Jura, M., Farihi, J., \& Zuckerman, B. 2007, ApJ, 663, 1285

Kanaan, A., Kepler, S. O., \& Winget, D. E. 2002, A\&A, 389, 896

Kaplan, D. L., Bhalerao, V. B., van Kerkwijk, M. H., et al. 2013, ApJ, 765, 158

Kenyon, S. J., \& Garcia, M. R. 1986, AJ, 91, 125

Kepler, S. O., Kleinman, S. J., Nitta, A., et al. 2007, MNRAS, 375, 1315

Kilic, M., Brown, W. R., Allende Prieto, C., et al. 2011, ApJ, 727, 3

Kilic, M., Brown, W. R., Allende Prieto, C., et al. 2012a, ApJ, 751, 141

Kilic, M., Brown, W. R., \& Hermes, J. J. 2013a, in ASP Conf. Ser. 467, 9th LISA Symposium, ed. G. Auger, P. Binétruy, \& E. Plagnol (San Francisco, CA: ASP), 47

Kilic, M., Gianninas, A., Brown, W. R., et al. 2013b, MNRAS, 434, 3582

Kilic, M., Hermes, J. J., Gianninas, A., et al. 2013c, arXiv:1310.6359

Kilic, M., Patterson, A. J., Barber, S., Leggett, S. K., \& Dufour, P. 2012b, MNRAS, 419, L59

Kilic, M., von Hippel, T., Leggett, S. K., \& Winget, D. E. 2006, ApJ, 646, 474

Klein, B., Jura, M., Koester, D., \& Zuckerman, B. 2011, ApJ, 741, 64

Klein, B., Jura, M., Koester, D., Zuckerman, B., \& Melis, C. 2010, ApJ, 709, 950

Koester, D., Rollenhagen, K., Napiwotzki, R., et al. 2005, A\&A, 432, 1025

Koester, D., \& Wilken, D. 2006, A\&A, 453, 1051

Liebert, J., Bergeron, P., \& Holberg, J. B. 2005, ApJS, 156, 47

Lodders, K. 2003, ApJ, 591, 1220
Marsh, T. R., Nelemans, G., \& Steeghs, D. 2004, MNRAS, 350, 113

Martin, D. C., Fanson, J., Schiminovich, D., et al. 2005, ApJL, 619, L1

Melis, C., Jura, M., Albert, L., Klein, B., \& Zuckerman, B. 2010, ApJ, 722, 1078

Morris, S. L., \& Naftilan, S. A. 1993, ApJ, 419, 344

Nather, R. E., \& Mukadam, A. S. 2004, ApJ, 605, 846

Panei, J. A., Althaus, L. G., Chen, X., \& Han, Z. 2007, MNRAS, 382, 779

Perryman, M. A. C., de Boer, K. S., Gilmore, G., et al. 2001, A\&A, 369,339

Rafikov, R. R. 2011a, ApJL, 732, L3

Rafikov, R. R. 2011b, MNRAS, 416, L55

Rafikov, R. R., \& Garmilla, J. A. 2012, ApJ, 760, 123

Schlegel, D. J., Finkbeiner, D. P., \& Davis, M. 1998, ApJ, 500, 525

Schmidt, G. D., Weymann, R. J., \& Foltz, C. B. 1989, PASP, 101, 713

Shporer, A., Kaplan, D. L., Steinfadt, J. D. R., et al. 2010, ApJL, 725, L200

Skrutskie, M. F., Cutri, R. M., Stiening, R., et al. 2006, AJ, 131, 1163

Stumpff, P. 1980, A\&AS, 41, 1

Thompson, S. E., \& Mullally, F. 2009, JPhCS, 172, 012081

Tremblay, P.-E., \& Bergeron, P. 2009, ApJ, 696, 1755

Tremblay, P.-E., Bergeron, P., Kalirai, J. S., \& Gianninas, A. 2010, ApJ, 712, 1345

Tremblay, P.-E., Ludwig, H.-G., Steffen, M., Bergeron, P., \& Freytag, B. 2011, A\&A, 531, L19

Tremblay, P.-E., Ludwig, H.-G., Steffen, M., \& Freytag, B. 2013a, A\&A, 552, A13

Tremblay, P.-E., Ludwig, H.-G., Steffen, M., \& Freytag, B. 2013b, A\&A, 559, A104

von Hippel, T., Kuchner, M. J., Kilic, M., Mullally, F., \& Reach, W. T. 2007, ApJ, 662,544

Wright, E. L., Eisenhardt, P. R. M., Mainzer, A. K., et al. 2010, AJ, 140, 1868

Wyder, T. K., Martin, D. C., Schiminovich, D., et al. 2007, ApJS, 173, 293

Zuckerman, B., Koester, D., Melis, C., Hansen, B. M., \& Jura, M. 2007, ApJ, 671,872

Zuckerman, B., Koester, D., Reid, I. N., \& Hünsch, M. 2003, ApJ, 596, 477 